\title{
Determination of Natural Frequency and Critical Velocity of Inclined Pipe Conveying Fluid under Thermal Effect by Using Integral Transform Technique
}

\author{
Jabbar Hussein Mohmmed*, Mauwafak Ali Tawfik, Qasim Abbas Atiyah \\ Department of Mechanical Engineering, University of Technology, Baghdad, Iraq \\ Received 28 June 2021; received in revised form 02 September 2021; accepted 03 September 2021 \\ DOI: https://doi.org/10.46604/aiti.2021.7988
}

\begin{abstract}
This study proposes an analytical solution of natural frequencies for an inclined fixed supported Euler-Bernoulli pipe containing the flowing fluid subjected to thermal loads. The integral transform technique is employed to obtain the spatial displacement-time domain response of the pipe-fluid system. Then, a closed-form analytical expression is presented. The effects of various geometric and system parameters on the vibration characteristics of pipe-fluid system with different flow velocities are discussed. The results illustrate that the proposed analytical solution agrees with the solutions achieved in previous works. The proposed model predicts that the pipe loses the stability by divergence with the increasing flow velocity. It is evident that the influences of inclination angle and temperature variation are dramatically increased at a higher aspect ratio. Additionally, it is demonstrated that the temperature variation becomes a more harmful effect than the internal fluid velocity on the stability of the pipe at elevated temperature.
\end{abstract}

Keywords: pipe conveying fluid, natural frequency, critical flow velocity, finite Fourier sine transform, Laplace transform

\section{Introduction}

Fluid-conveying pipes are very important parts in many engineering structures and have many potential applications in industry. Practical applications include chemical plants, nuclear reactors, oil and gas industries, heat exchangers, fuel lines of transportations, monitoring and controlling tubes, marine risers, and so on. Other applications in engineering devices involve jet pumps, some kinds of valves and parts of hydraulic machinery, and submarine systems, etc. Hemodynamics and the pulmonary and urinary systems also may be including some applications of the pipes conveying fluid [1-2]. The function of these pipes is very important and can be considered the blood circulation of human body. The fluid flowing inside these pipes influences the dynamic behaviors of the piping systems, which play a significant role in the stability and the performance of the systems. The influences of the fluid and structure interaction usually lead to the vibration of the pipe and even the rupture of the pipe.

Thus, a convenient knowledge of the dynamic behavior of these types of systems is essential and has motivated many scholars over the last six decades. Extensive investigations have been executed on the vibration analysis of the fluid-conveying pipes subjected to various end and loading conditions. The first correct motion equation of the structure-fluid coupling vibration for a pipe containing flowing fluid was proposed by Housner [3]. Thereafter, the vibration characteristics of pipe-fluid system have been increasingly studied by several other scholars. Stein and Tobriner [4] discovered that the internal pressure of flowing fluid has inverse effect on the natural frequency of the system. Païdoussis [5] further investigated the dynamic behavior of conservative system, predicting that the system may be undergoing the flexural oscillatory instability by divergence at a certain high flow speed, and that at higher flow speed the system can be subjected to coupled-mode flutter. Plaut and Huseyin [6], Hatfield et al. [7], and Lesmez et al. [8] further analyzed the plain and U-bend piping systems.

* Corresponding author. E-mail address:10493@uotechnology.edu.iq 
Nowadays, with the existence of larger pipeline systems, the demand for inclined pipes have been grown due to their special conditions, and it is therefore worthy being concerned about. Moreover, these pipes can be possessed various aspect ratios of length to external diameter. A better understanding of the influence of the aspect ratio and inclination angle on the dynamic behavior of a pipe is very essential to design thin components in different industries. Additionally, when the pipes are designed and analyzed, it is very important to take account of the working conditions which can affect the dynamic behavior and integrity of such components. One of the important examples of such operating conditions is the temperature variation, which is undergone during the conveyance of fluid in pipes. The pipes in such cases suffer the compressive stresses resulting from the thermal effect in addition to those generated by the fluid flowing through the pipe. Such pipes can experience buckling and show the complicated dynamical behavior related to their divergence even at a very low internal flow velocity of fluid [9]. Consequently, temperature is one of the main concerns among other factors and operating conditions in designing slender structures like the pipeline systems containing flowing fluid.

Recently, Qian et al. [9] studied the effects of both linear and non-linear stress-temperature cases on the stability of pipe containing flowing fluid. Zhao et al. [10] investigated the stability of the simply supported pipes induced by pulsating fluid velocity and thermal loading. Kukla [11] studied the temperature distribution and lateral vibration of beam induced by external heating source. Blandino and Thornton [12] performed a study about the vibration characteristic of an internally heated beam. Alfosail et al. [13] numerically evaluated the dynamic behavior of inclined risers. Gan et al. [14] investigated the stability of an inclined cantilevered pipe containing flowing fluid. Yang and Wang [15] analyzed the dynamic behavior of an inclined beam induced by moving loads.

From the literature review, it is observed that no previous works have been reported on the response of pipes due to a combination effect of internal fluid, inclination angle, aspect ratio, and thermal loads. Furthermore, it is noted that most of the above-mentioned analyses have been based on numerical or approximate approaches in tackling linear and non-linear dynamics issues of these slender structures. This approximation is valid only for small values of the applied loads and self-weight. It has been found that there are quite a few articles that use analytical approaches to analyze the dynamic problems of the pipes conveying fluid. An analytical solution is very important for the improvements and verifications of effective applied numerical simulation tools. To address the lack of research in this aspect, the analytic solution based on integral transform technique (ITT) approach (via the combination of finite Fourier sine and Laplace transforms) is adopted in this work to analyze the dynamic behavior of an inclined and doubly fixed supported pipe subjected to the thermal loads. Firstly, the frequency-domain response of the pipe conveying fluid is obtained. Then, the corresponding inverse transforms on the displacement frequency-domain responses of the pipe are conducted to obtain the spatial displacement time-domain responses. Moreover, the effects of changing the aspect ratio, inclination angle, and temperature variation on the natural frequencies, as well as the critical velocities of the pipes conveying fluid under different fluid speeds are evaluated.

This work is structured as follows. Section 1 introduces the problem under study. Section 2 presents the methods used to solve motion equations. Section 3 provides the model description and solution procedure of governing equations. In section 4 , results are analyzed and discussed. Finally, the paper ends in section 5 with the summary and conclusions of the current work.

\section{Methods}

\subsection{Laplace transform}

Laplace transform is a linear transform that is widely applied in the dynamic problems of structure. It provides easy and powerful means to transform partial differential equations into ordinary differential equations, or transform ordinary differential equations into integral form equations, which make them much easier to be solved. Laplace transform directly obtains the solution of differential equations with certain boundary values without determining the general solution first 
[16-17]. Many successful applications of Laplace transform were done to study the dynamic behavior of the components with and without external forces [18-19]. Assume that $f_{(t)}$ is a real function of time $t$, which is defined in the real domain [0, $\left.+\infty\right)$, the Laplace transform and its inversion are defined by:

$$
\begin{aligned}
& \psi_{(s)}=L\left[f_{(t)}\right]=\int_{0}^{\infty} f_{(t)} e^{-s t} d t \\
& f_{(t)}=L^{-1}\left[\psi_{(s)}\right]=\frac{1}{2 \pi i} \int_{\mathcal{E}-i \infty}^{\varepsilon+i \infty} \psi_{(s)} e^{s t} d s
\end{aligned}
$$

where $L$ is Laplace transform, $L^{-1}$ is the inverse Laplace transform, $s$ represents a complex number in the Laplace domain, and $s_{i}(\mathrm{i}=1,2,3, \ldots)$ are the singularities of $\psi_{(s)}$.

\subsection{Finite Fourier sine transform}

The Fourier transform method was utilized into the field of structural dynamics by many researchers [20-21]. Fourier transform is helpful in solving the differential and partial differential equations of non-periodic functions. It can be applied to solve some boundary-value and initial-value problems for partial differential equations. Fourier transform is usually used in order to reduce the order of differential equations. It can be employed to convert the infinite degree of freedom systems into a superimposed double or single degree of freedom system. The finite sine Fourier transform for a function of $f_{(x)}$, which is a real function of coordinate $x$ in space $(0 \leq x \leq 1)$, can be represented as follows [22].

$$
\begin{aligned}
& F_{s}\left[f_{(x)}\right]=\int_{0}^{1} f_{(x)} \sin n \pi x d x=f_{(n)} \\
& F_{s}^{-1}\left[f_{(n)}\right]=2 \sum_{n=1}^{\infty} f_{(n)} \sin n \pi x=f_{(x)}
\end{aligned}
$$

where $F_{S}$ is Fourier transform, $F_{S}^{-1}$ is the inverse Fourier transform, and $n$ is an integer.

\section{Model Description}

\subsection{Governing equation}

The system considered in the present investigation comprises a linear elastic, clamped ends, and the inclined pipes made of polypropylene-random (PP-R) material with total length $(L)$, inner and outer diameter $\left(D_{i}\right.$ and $\left.D_{o}\right)$, thickness $(t)$, cross-sectional area $\left(A_{p}\right)$, mass per unit length $\left(m_{p}\right)$, Young's modulus $(E)$, and moment of inertia $(I)$. The fluid passing through the pipe has a mass per unit length $m_{f}$ with an axial internal fluid flowing velocity $v$. $A$ represents the cross-sectional area of the flow and is assumed to be a constant. The fluid has no compression properties and has no viscosity. Unlike the discrete systems (simple or torsional), the continuous systems, like pipe or beam, possess an infinite number of degrees of freedom that make them more difficult to be treated. Hence, many assumptions were made to simplify the working process with these systems [23-24]. Figs. 1 and 2 present the geometry and Cartesian coordinate system, assuming that the origin of the ( $x, y, z)$ framework is placed in the space at the left end of the pipe. The properties of pipe and fluid and the numeric parameters considered in this study are presented in Table 1 .

The current study focuses on finding the natural frequencies and critical velocities of axial flow fluid-carrying pipes under different conditions. Since the non-planar motion (three-dimensional motion) does not affect the values of the natural frequencies or critical velocities, it is neglected and the planar motion (two-dimensional motion) of the pipes is adopted. Fig. 3 illustrates the fluid and pipe elements, and shows all the forces acting on the system. 
Table 1 Numeric values of used parameters

\begin{tabular}{|c|c|}
\hline Specification & Value \\
\hline Material & PP-R \\
\hline Fluid & Water \\
\hline Outer diameter $D_{o}(\mathrm{~m})$ & 0.025 \\
\hline Inner diameter $D_{i}(\mathrm{~m})$ & 0.018 \\
\hline Thickness $t(\mathrm{~m})$ & 0.0035 \\
\hline Length $L(\mathrm{~m})$ & $0.5-1.25$ \\
\hline Aspect ratio (length to outer diameter $) L^{\prime} D_{o}$ & $(20-50) D_{o}$ \\
\hline & 0.8 at $25^{\circ} \mathrm{C}$ \\
\cline { 2 - 2 } Modulus of elasticity $E(\mathrm{GPa})$ & 0.38 at $50^{\circ} \mathrm{C}$ \\
\cline { 2 - 2 } & 0.23 at $70^{\circ} \mathrm{C}$ \\
\hline Density of pipe $\rho_{p}\left(\mathrm{Kg} / \mathrm{m}^{3}\right)$ & 909 \\
\hline Density of fluid $\rho_{f}\left(\mathrm{Kg} / \mathrm{m}^{3}\right)$ & 1000 \\
\hline Coefficient of expansion $\alpha(1 / \mathrm{K})$ & $0.3 \times 10^{-4}$ \\
\hline
\end{tabular}
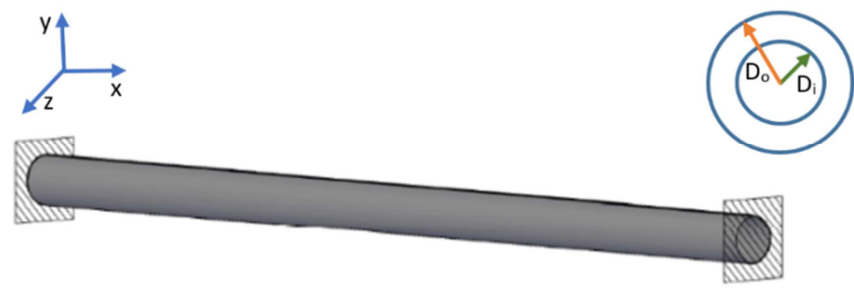

Fig. 1 Three-dimension geometry of the clamped-end pipeline

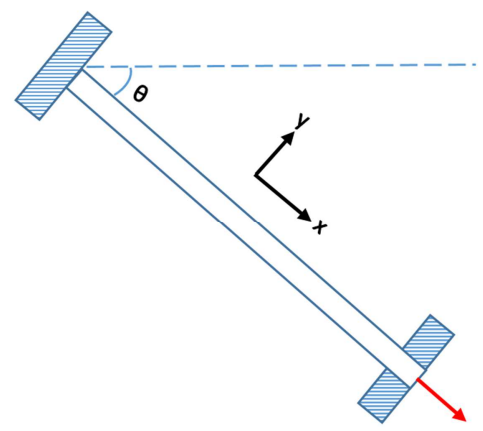

Fig. 2 Schematic of the clamped-end inclined pipe

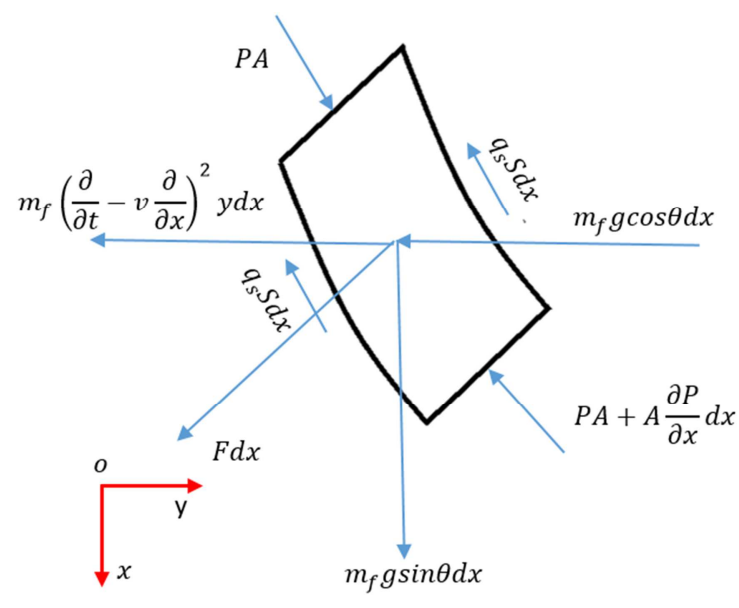

(a) Fluid element

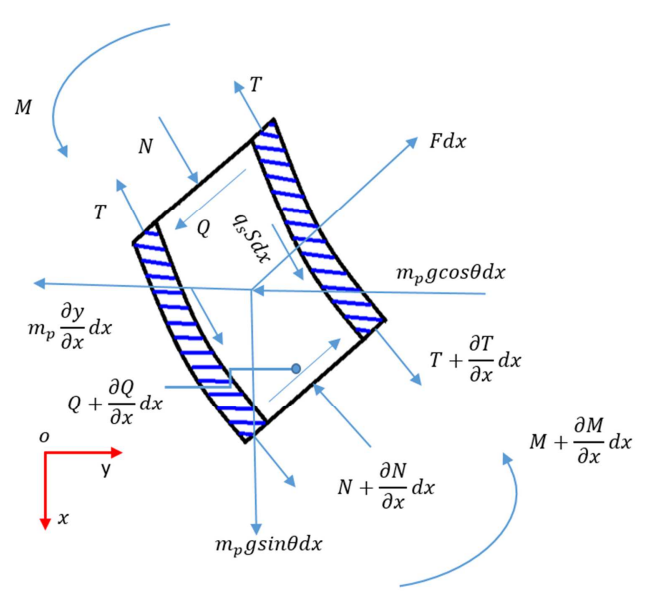

(b) Pipe element

Fig. 3 Free-body diagram of the fluid and pipe element

To simplify the derivation process, some assumptions are adopted: considering the vertical motion (y-axis) of the pipe; ignoring internal damping (viscoelastic effect), dissipation, external tension, and internal pressurization; assuming a small deformation; treating the pipe as an Euler-Bernoulli beam model. Then, using the Newtonian method by balancing forces on 
the fluid and pipe elements in the transverse and longitudinal directions, and after some substitutions processes and mathematical calculations, the following motion equation of the heated and inclined pipe can be derived (more details about the assumptions and derivation process can be found in the work of Mohmmed et al. [25]):

$$
\begin{aligned}
& E I \frac{\partial^{4} y}{\partial x^{4}}-\left(m_{f}+m_{p}\right)(L-x) g \sin \theta \frac{\partial^{2} y}{\partial x^{2}}+m_{f} v^{2} \frac{\partial^{2} y}{\partial x^{2}}+N \frac{\partial^{2} y}{\partial x^{2}}+\left(m_{f}+m_{p}\right) g \sin \theta \frac{\partial y}{\partial x}+\left(m_{f}+m_{p}\right) g \cos \theta \\
& +2 m_{f} v \frac{\partial^{2} y}{\partial x \partial t}+\left(m_{f}+m_{p}\right) \frac{\partial^{2} y}{\partial^{2} t}=0
\end{aligned}
$$

where $E I$ is the flexural rigidity of the pipe; $g$ is gravitational constant; $\theta$ is the inclination angle of the pipe with horizontal axis; $q_{s}$ represents internal shear stress; $p$ is internal fluid pressure; $S$ is the internal perimeter (internal section circumference) of the pipe; $F$ is the transverse force between pipe wall and fluid (per unit length); $T$ represents longitudinal tension. $M$ is bending moment; $Q$ represents the transverse shear force in the pipe; $y(x, t)$ is the lateral displacement of the pipe; $x$ and $t$ are the axial coordinate and time, respectively. $N$ represents the thermal load in the pipe due to temperature variation and can be calculated as: $N=\alpha E A_{p} \Delta T$, where $\alpha$ is thermal expansion coefficient $\left(1 /{ }^{\circ} \mathrm{C}\right)$ and $L$ is pipe length $(\mathrm{m}) . \Delta T=\left(T_{p t}\right)_{x}-T_{i}$ is the temperature change $\left({ }^{\circ} \mathrm{C}\right) . T_{i}$ is the initial temperature of the pipe $\left({ }^{\circ} \mathrm{C}\right) .\left(T_{p t}\right)_{x}$ is the instantaneous temperature of the pipe after the time $\left({ }^{\circ} \mathrm{C}\right)$.

The terms of Eq. (5) can be described as follows: the first component represents the acceleration resulting from the restoring force, and the second and third components represent the accelerations due to the gravity force and the centrifugal force respectively. After that, the forth component associates with the acceleration due to the thermal force caused by the temperatures variation, then the fifth and sixth components illustrate the static force because of the gravity effect. The seventh component stands for the acceleration due to the Coriolis forces, following the acceleration because of the system's inertia force. Rearranging Eq. (5) gives:

$$
\begin{aligned}
& E I \frac{\partial^{4} y}{\partial x^{4}}-\left[\left(m_{f}+m_{p}\right)(L-x) g \sin \theta-N-m_{f} v^{2}\right] \frac{\partial^{2} y}{\partial x^{2}}+\left(m_{f}+m_{p}\right) g \sin \theta \frac{\partial y}{\partial x}+\left(m_{f}+m_{p}\right) g \cos \theta+2 m_{f} v \frac{\partial^{2} y}{\partial x \partial t} \\
& +\left(m_{f}+m_{p}\right) \frac{\partial^{2} y}{\partial^{2} t}=0
\end{aligned}
$$

Eq. (6) can be rewritten in non-dimensional form as follows:

$$
\frac{\partial^{4} \phi}{\partial \zeta^{4}}-\left[(1-\zeta) G \sin \theta-\bar{N}-U^{2}\right] \frac{\partial^{2} \phi}{\partial \zeta^{2}}+G \sin \theta \frac{\partial \phi}{\partial \zeta}+G \cos \theta+2 \beta^{1 / 2} U \frac{\partial^{2} \phi}{\partial \zeta \partial t}+\frac{\partial^{2} \phi}{\partial^{2} t}=0
$$

where

$$
\begin{aligned}
& \phi=\frac{y}{L} \\
& \zeta=\frac{x}{L} \\
& \beta=\frac{m_{f}}{m_{f}+m_{p}} \\
& G=\frac{(m+M) g L^{3}}{E I} \\
& U=\left(\frac{m_{f}}{E I}\right)^{\frac{1}{2}} v L
\end{aligned}
$$




$$
\begin{aligned}
& \bar{t}=\frac{1}{L^{2}}\left(\frac{E I}{m_{f}+m_{p}}\right)^{\frac{1}{2}} \\
& \bar{N}=\frac{N L^{2}}{E I} \\
& \phi^{\prime \prime \prime \prime}-\left[(1-\zeta) G \sin \theta-\bar{N}-U^{2}\right] \phi^{\prime \prime}+G \sin \theta \phi^{\prime}+2 \beta^{1 / 2} U \ddot{\phi}^{\prime}+\ddot{\phi}=-G \cos \theta
\end{aligned}
$$

\subsection{Solution procedure}

In order to discrete Eq. (15) and transform it to an ordinary differential equation, the term $\eta(\zeta, \tau)$ is used to decompose the equation into space and time as follows:

$$
\phi(\zeta, \bar{t})=\Gamma(\zeta) \Lambda(\bar{t})
$$

where $\Lambda(\bar{t})$ the generalized coordinate of the system and $\Gamma(\zeta)$ is a trial/comparison function satisfying both the geometrical and natural boundary conditions.

The pipe end boundary conditions considered in the current study is clamped-clamped support, which can be symbolized as C-C. The boundary conditions at the edges can be expressed as: clamped $(\zeta=0)-$ clamped $(\zeta=1), \mathrm{C}-\mathrm{C}$ at $\zeta=0, \Gamma=0$, and $\partial \Gamma / \partial \zeta=0 ;$ at $\zeta=1, \Gamma=0$, and $\partial \Gamma / \partial \zeta=0$. Substituting Eq. (16) into Eq. (15) gives:

$$
\begin{aligned}
& \Gamma^{\prime \prime \prime \prime}(\zeta) \Lambda(\bar{t})-\left[(1-\zeta) G \sin \theta-\bar{N}-U^{2}\right] \Gamma^{\prime \prime}(\zeta) \Lambda(\bar{t})+G \sin \theta \Gamma^{\prime}(\zeta) \Lambda(\bar{t})+2 \beta^{1 / 2} U \Gamma^{\prime}(\zeta) \dot{\Lambda}(\bar{t})+\Gamma(\zeta) \ddot{\Lambda}(\bar{t}) \\
& =-G \cos \theta
\end{aligned}
$$

To solve Eq. (17), ITTs are used in this work. First, based on Eq. (1), by assuming zero initial conditions case, Laplace transform is introduced to suppress the time dependency.

$$
\begin{aligned}
& \Gamma^{\prime \prime \prime \prime}(\zeta) \Lambda(s)-\left[(1-\zeta) G \sin \theta-\bar{N}-U^{2}\right] \Gamma^{\prime \prime}(\zeta) \Lambda(s)+G \sin \theta \Gamma^{\prime}(\zeta)+2 \beta^{1 / 2} U s \Gamma^{\prime}(\zeta) \Lambda(s)+s^{2} \Gamma(\zeta) \Lambda(s) \\
& =-\frac{G}{s} \cos \theta
\end{aligned}
$$

Next, for reducing the difficulty of solving Eq. (18), a finite Fourier sine transform is introduced to convert the infinite degree of freedom pipe-fluid system into a superimposed double degrees of freedom system. Thus, by using Eq. (3), finite Fourier sine transform with regard to spatial (space) coordinate $\Gamma$ is conducted on Eq. (18), and the following equation can be obtained:

$$
\begin{aligned}
& \Lambda(s) \int_{0}^{1} \Gamma^{\prime \prime \prime \prime}(\zeta) \sin n \pi \zeta d \zeta-\left(G \sin \theta-\bar{N}-U^{2}\right) \Lambda(s) \int_{0}^{1} \Gamma^{\prime \prime}(\zeta) \sin n \pi \zeta d \zeta+G \sin \theta \Lambda(s) \int_{0}^{1} \zeta \cdot \Gamma^{\prime \prime}(\zeta) \sin n \pi \zeta d \zeta \\
& +G \sin \theta \Lambda(s) \int_{0}^{1} \Gamma^{\prime}(\zeta) \sin n \pi \zeta d \zeta+2 \beta^{1 / 2} U s \Lambda(s) \int_{0}^{1} \Gamma^{\prime}(\zeta) \sin n \pi \zeta d \zeta+s^{2} \Lambda(s) \int_{0}^{1} \Gamma(\zeta) \sin n \pi \zeta d \zeta \\
& =-\frac{G}{s} \cos \theta \int_{0}^{1}(1) \sin n \pi \zeta d \zeta
\end{aligned}
$$

$\Gamma(\zeta)$ is beam Eigen function and varies according to boundary condition for the clamped supported case:

$$
\Gamma(\zeta)=\cosh \left(\lambda_{r} \zeta\right)-\cos \left(\lambda_{r} \zeta\right)-\left[\frac{\cosh \left(\lambda_{r} \mathrm{~L}\right)-\cos \left(\lambda_{r} \mathrm{~L}\right)}{\sinh \left(\lambda_{r} \mathrm{~L}\right)-\sin \left(\lambda_{r} \mathrm{~L}\right)}\right]\left[\sinh \left(\lambda_{r} \zeta\right)-\sin \left(\lambda_{r} \zeta\right)\right]
$$




$$
\Gamma(\zeta)=\cosh \left(\lambda_{r} \zeta\right)-\cos \left(\lambda_{r} \zeta\right)-\left[\frac{\sinh \left(\lambda_{r} \mathrm{~L}\right)+\sin \left(\lambda_{r} \mathrm{~L}\right)}{\cosh \left(\lambda_{r} \mathrm{~L}\right)-\cos \left(\lambda_{r} \mathrm{~L}\right)}\right]\left[\sinh \left(\lambda_{r} \zeta\right)-\sin \left(\lambda_{r} \zeta\right)\right]
$$

where $\lambda_{r}$ is the root of the equation $\cosh \left(\lambda_{r} \mathrm{~L}\right) \cos \left(\lambda_{r} \mathrm{~L}\right)$.

The using of terms included in Eq. (20) or (21) for fixed support may contain some difficulty and complexity in determining the integral transformation for each term in Eq. (19). Thus, for simplification, a polynomial function can be applied for this type of support system:

$$
\Gamma(\zeta)=a_{0}+a_{1} \zeta+a_{2} \zeta^{2}+a_{3} \zeta^{3}+a_{4} \zeta^{4}
$$

Applying the boundary conditions of C-C gives:

$$
\Gamma(\zeta)=\left(\zeta^{4}-2 \zeta^{3}+\zeta^{2}\right) a_{4}
$$

Using orthogonal functions gives:

$$
a_{4}=3 \sqrt{70}\left[\sqrt{\frac{1}{a^{5}\left(70 a^{4}-315 a^{3}+540 a^{2}-420 a+126\right)}}\right]
$$

By substituting $a=1$ in Eq. (21), $a_{4}=25.20$ can be obtained for the first mode. By substituting Eq. (23) in Eq. (19), performing the integral transformation, and reorganizing and combining similar terms, the following equation is yielded:

$$
\begin{aligned}
& \left\{\begin{array}{l}
s^{2}\left\{\begin{array}{l}
\frac{24}{n^{5} \pi^{5}}\left[1+(-1)^{n+1}\right]- \\
\frac{1}{n^{3} \pi^{3}}\left[1+(-1)^{n+1}\right]
\end{array}\right\}+s\left\{2 \beta^{1 / 2} U\left\{\frac{12}{n^{3} \pi^{3}}\left[1+(-1)^{n}\right]\right\}\right\}+\frac{24}{n \pi}\left[1+(-1)^{n+1}\right]-\left(\bar{N}+U^{2}\right) \\
\left\{\begin{array}{l}
\frac{2}{n \pi}\left[1+(-1)^{n+1}\right]- \\
\frac{24}{n^{3} \pi^{3}}\left[1+(-1)^{n+1}\right]
\end{array}\right\}+G \sin \theta\left\{\begin{array}{l}
\frac{2}{n \pi}\left[1+(-1)^{n+1}\right]-\frac{24}{n^{3} \pi^{3}}\left[1+(-1)^{n+1}\right]+\frac{24}{n^{3} \pi^{3}} \\
{\left[1+2(-1)^{n}\right]+\frac{2(-1)^{n+1}}{n \pi}+\frac{12}{n^{3} \pi^{3}}\left[1+(-1)^{n}\right]}
\end{array}\right\} \\
=-\frac{1}{a_{4}} \frac{G}{s} \cos \theta \cdot 1(f)
\end{array}\right\} \Lambda(s)
\end{aligned}
$$

where

$$
\int_{0}^{1}(1) \sin n \pi \zeta d \zeta=\frac{\left[1+(-1)^{n+1}\right]}{n \pi}=1(f)
$$

Eq. (25) can be simplified by assuming:

$$
\begin{aligned}
& Z_{f 1}=2 \beta^{1 / 2} U\left[\frac{12}{n^{3} \pi^{3}}\left(1+(-1)^{n}\right)\right] \\
& Z_{f 2}=\frac{24}{n \pi}\left[1+(-1)^{n+1}\right]-\left(\bar{N}+U^{2}\right)\left\{\begin{array}{l}
\frac{2}{n \pi}\left[1+(-1)^{n+1}\right]- \\
\frac{24}{n^{3} \pi^{3}}\left[1+(-1)^{n+1}\right]
\end{array}\right\}+G \sin \theta\left\{\begin{array}{l}
\frac{2}{n \pi}\left[1+(-1)^{n+1}\right]-\frac{24}{n^{3} \pi^{3}}\left[1+(-1)^{n+1}\right]+\frac{24}{n^{3} \pi^{3}} \\
{\left[1+2(-1)^{n}\right]+\frac{2(-1)^{n+1}}{n \pi}+\frac{12}{n^{3} \pi^{3}}\left[1+(-1)^{n}\right]}
\end{array}\right\}
\end{aligned}
$$




$$
\therefore\left\{s^{2}\left\{\frac{24}{n^{5} \pi^{5}}\left[1+(-1)^{n+1}\right]-\frac{1}{n^{3} \pi^{3}}\left[1+(-1)^{n+1}\right]\right\}+Z_{f 1} s+Z_{f 2}\right\} \Lambda(s)=-\frac{1}{a_{4}} \frac{G}{s} \cos \theta .1(f)
$$

Additionally, the following equations can be assumed:

$$
\begin{aligned}
& \varphi_{f 1}=\frac{Z_{f 1}}{\frac{24}{n^{5} \pi^{5}}\left[1+(-1)^{n+1}\right]-\frac{1}{n^{3} \pi^{3}}\left[1+(-1)^{n+1}\right]} \\
& \varphi_{f}^{2}=\frac{Z_{f 2}}{\frac{24}{n^{5} \pi^{5}}\left[1+(-1)^{n+1}\right]-\frac{1}{n^{3} \pi^{3}}\left[1+(-1)^{n+1}\right]} \\
& \therefore \Lambda(s)=\frac{-\frac{1}{a_{4}} \frac{G}{s} \cos \theta \cdot 1(f)}{\frac{24}{n^{5} \pi^{5}}\left[1+(-1)^{n+1}\right]-\frac{1}{n^{3} \pi^{3}}\left[1+(-1)^{n+1}\right]} \\
& s^{2}+\varphi_{f 1} s+\varphi_{f}^{2}
\end{aligned}
$$

By applying the Fourier-Laplace inversion, the spatial displacement time-domain response (dynamic response $\phi$ ) for the inclined fixed supported pipe conveying fluid can be given:

$$
\begin{aligned}
& \phi(\zeta, \bar{t})=\Gamma(\zeta) \frac{-G \cos \theta \cdot \mathrm{F}_{f}(\bar{t})}{a_{4}\left(\frac{24}{n^{4} \pi^{4}}-\frac{2}{n^{2} \pi^{2}}\right)} \\
& \mathrm{F}_{f}(\bar{t})=\left[\frac{1}{\alpha_{f 1} \alpha_{f 2}}+\frac{1}{\alpha_{f 1} \alpha_{f 2}\left(\alpha_{f 2}-\alpha_{f 1}\right)}\left(\alpha_{f 1} e^{-\alpha_{f 2} \bar{t}}-\alpha_{f 2} e^{-\alpha_{f 1} \bar{t}}\right)\right] \\
& \alpha_{f 1}=\frac{\varphi_{f 1}}{2}+i \sqrt{\varphi_{f}^{2}-\frac{\varphi_{f 1}{ }^{2}}{4}} \\
& \alpha_{f 2}=\frac{\varphi_{f 1}}{2}-i \sqrt{\varphi_{f}^{2}-\frac{\varphi_{f 1}{ }^{2}}{4}}
\end{aligned}
$$

By isolating characteristic equations, the dimensionless fundamental natural frequency $\left(\omega_{n}\right)$ and its complimentary value $\left(\omega_{m}\right)$ are:

$$
\begin{aligned}
& \omega_{n}^{2}=\left(\frac{\varphi_{f 1}}{2}\right)^{2} \mp\left(\varphi_{f}^{2}-\frac{\varphi_{f 1}^{2}}{4}\right) \Rightarrow \omega_{n}=\sqrt{\varphi_{f}^{2}-\frac{\varphi_{f 1}{ }^{2}}{4}} \\
& \omega_{m}=\sqrt{-\varphi_{f}^{2}}
\end{aligned}
$$

The fundamental natural frequency $\omega_{n}$ is adopted in this work. The aforementioned equations are solved for each value of variables by using MATLAB 2019b software program to determine the fundamental natural frequency of the system. The simplified flow chart is illustrated in Fig. 4. 


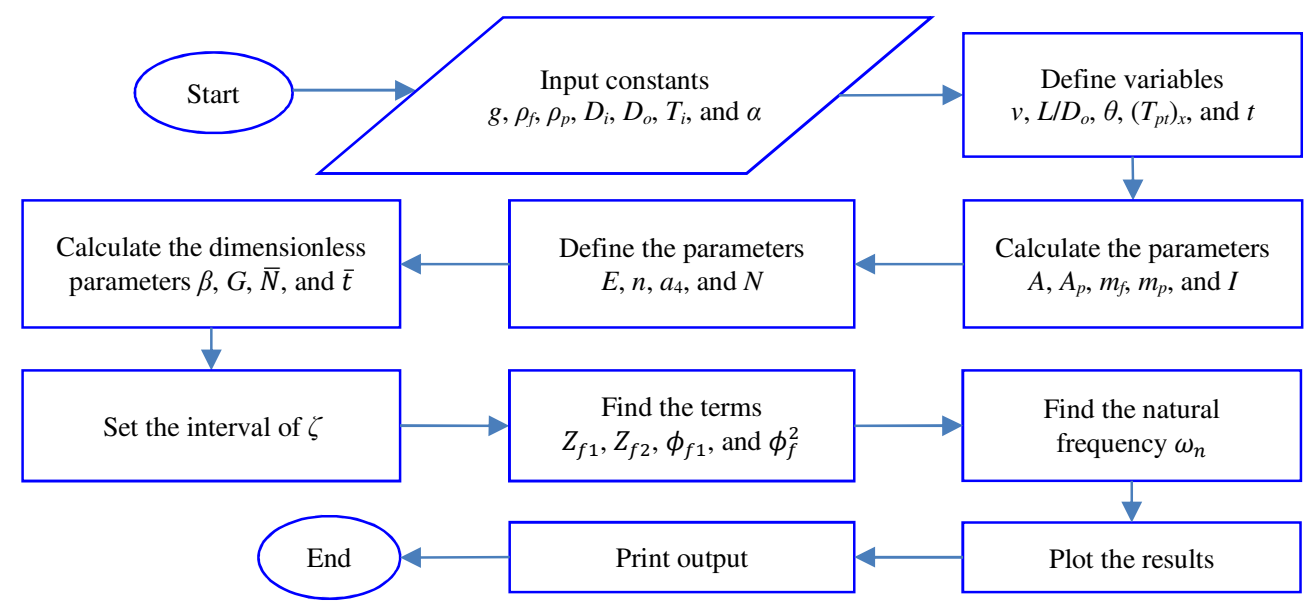

Fig. 4 The flowchart for MATLAB code

\section{Results and Discussion}

\subsection{Validation of analytical model}

To validate the analytical calculation method proposed in this work, the fundamental natural frequency is calculated at stationary fluid case $(U=0)$ and at fluid velocities $U=0.5, U=1$, and $U=1.5$, respectively, and compared with those in the work of $\mathrm{Ni}$ et al. [26] and Liang et al. [27]. Table 2 presents the geometry and material properties used in this comparison, and lists the obtained results. It is clear from Table 3 that the relative errors between the results in current study and those in Refs. [26-27] are less than $2.46 \%$. These little differences may be attributed to the using of different models in the two references. In Refs. [26-27], the pipe is modeled according to Timoshenko theory; in the current work the Euler-Bernoulli beam is adopted. It can be concluded from this comparison that the applied integral transform method (via the combination of the finite Fourier sine transform and Laplace transform) gives quite reliable results provided that the fluid flow velocity is moderate.

Table 2 Parameters of pipe and fluid

\begin{tabular}{|c|c|c|c|c|}
\hline Young's modulus & Pipe size & Pipe length & Pipe density & Fluid density \\
\hline \multirow{2}{*}{$210 \mathrm{GPa}$} & $D_{o}=324 \mathrm{~mm}$ & $32 \mathrm{~m}$ & $8200 \mathrm{~kg} / \mathrm{m}^{3}$ & $908.2 \mathrm{~kg} / \mathrm{m}^{3}$ \\
\cline { 2 - 2 } & $D_{i}=292 \mathrm{~mm}$ & & \\
\hline
\end{tabular}

Table 3 Comparison of dimensionless natural frequency of the fixed supported pipe with different fluid velocities

\begin{tabular}{|c|c|c|c|c|c|}
\hline \multirow[b]{2}{*}{ Fluid velocity } & Current value & Ref. [26] & \multirow{2}{*}{ Relative error $\%$} & Ref. [27] & \multirow{2}{*}{ Relative error \% } \\
\hline & $\omega_{\mathrm{n}}$ & $\omega_{\mathrm{n}}$ & & $\omega_{\mathrm{n}}$ & \\
\hline$U=0$ & 22.3087 & 22.2597 & 0.22 & 22.42 & 0.49 \\
\hline$U=0.5$ & 22.2534 & 22.0910 & 0.73 & 22.27 & 0.07 \\
\hline$U=1$ & 22.0864 & 21.9224 & 0.74 & 22.11 & 0.10 \\
\hline$U=1.5$ & 21.8053 & 21.4165 & 1.81 & 21.28 & 2.46 \\
\hline
\end{tabular}

\subsection{Natural frequency}

In the current study, the natural frequency and stability of an elastic, inclined, and doubly fixed supported pipe conveying a Newtonian and incompressible fluid at different temperature are investigated. The natural frequencies of the pipe are analytically calculated based on Eq. (37). The values of the system parameters used to obtain the results are listed in Table 1. To connect the subcritical, critical, and post-critical vibratory behaviors, the continuity profile of the real and imaginary components of pipe-fluid system's natural frequencies (instead of separated profile as widely observed in the literature) is adopted in this study by plotting the natural frequencies in absolute values against the internal flow velocity. 
The natural frequency of the pipe-fluid system is demonstrated in Figs. 5-9. Two zones are identified by observing the profile of fundamental natural frequency with respect to the influence of flow speed in Figs. 5 and 8 ; where the two zones illustrate the divergence case when the flow is lower and higher than the critical points of flow. In general, the results show that the fundamental natural frequency of the system is affected by factors such as fluid velocity, and tends to reduce as the velocity of fluid flowing within the pipe increases. With the increasing fluid velocity, the fundamental natural frequency of the pipe vanishes, and then the divergence instability happens. The corresponding fluid velocity, at which the fundamental natural frequency vanishes, is named critical velocity. This is because the increase of the velocity of fluid flowing inside the pipe leads to the weakness of the pipe stiffness. Almost similar conclusions in the subcritical fluid velocity range (1st zone) were observed in the work of $\mathrm{Ni}$ et al. [26] and in the supercritical regime (2nd zone) in the work of Adelaja [28] and Olunloyo et al. [29], while for the whole spectrum results the same trend was found in the work of Plaut [30].

Fig. 5 presents the effect of different aspect ratio (length to diameter ratio) on the natural frequency. It is clear that the natural frequencies of the fluid-conveying pipe decrease with the increase of length to diameter ratio. Similarly, the critical fluid velocity decreases with the increase of the aspect ratio. This suggests that the pipe-fluid system will hastily reach divergence instability situation with higher aspect ratio. The reason is that the higher length to diameter ratio leads to the increase of the pipe weight resulting in the weakness of its stuffiness. The same trend was observed in the work of Liang [27] and Olunloyo et al. [31] with the increase of the pipe length. Meanwhile, by observing Fig. 5, it is evident that the level of reduction in the magnitude of natural frequencies and critical velocity for the same aspect ratio reduces with the increase of the support angle. At the same time, by observing Fig. 5, it is clear that the level of reduction in the value of the natural frequency and critical velocity decreases for the same aspect ratio with the increase of the inclination angle.

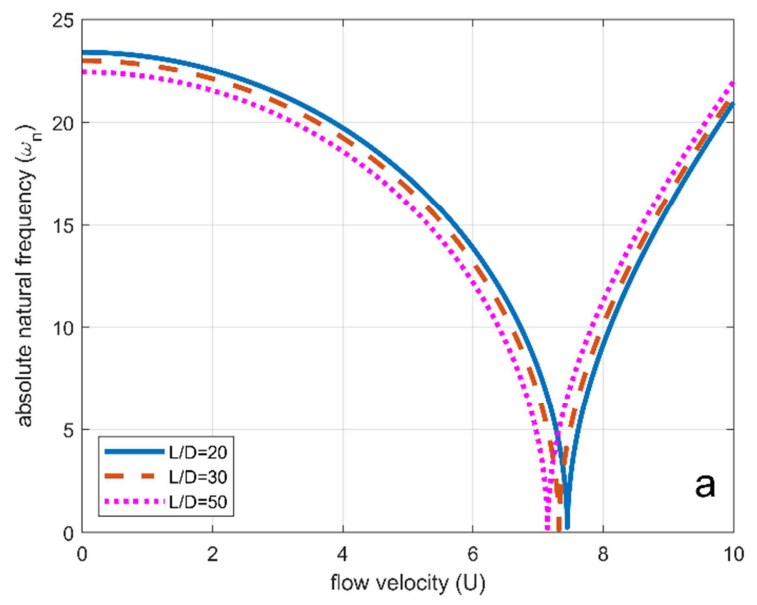

(a) $\theta=0^{\circ}$

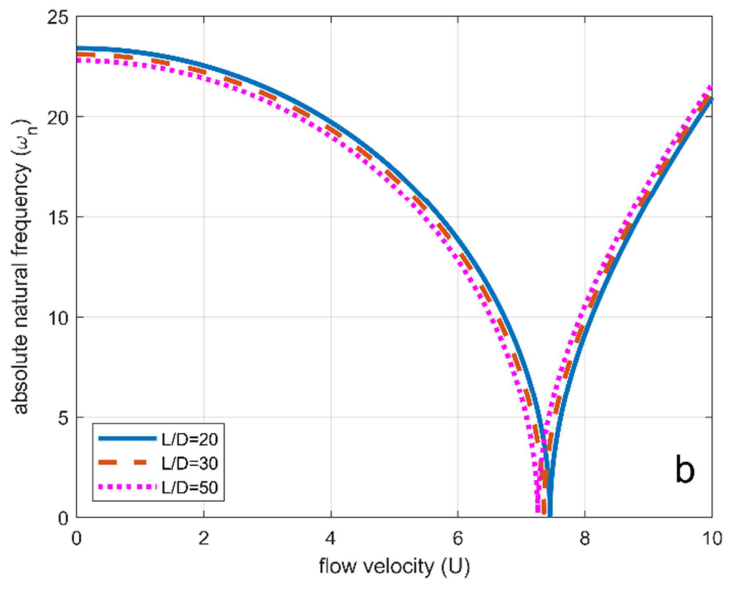

(b) $\theta=15^{\circ}$

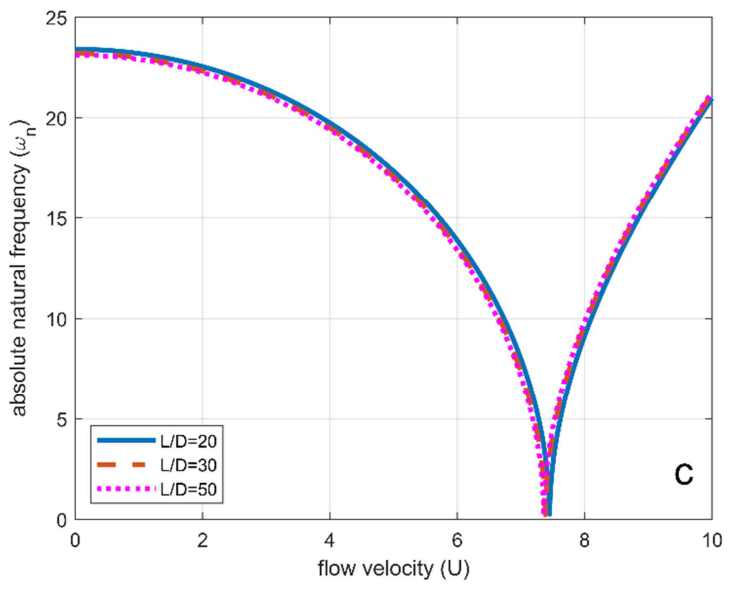

(c) $\theta=30^{\circ}$

Fig. 5 The natural frequency value versus fluid velocity with different aspect ratios for the case of $T=25^{\circ} \mathrm{C}$ and $n=1$ 
The effect of the pipe's inclination angle and aspect ratio on the fundamental natural frequency of the structure is studied further in Figs. 6 and 7 respectively. In general, the results indicate the increase of natural frequencies with the increase of the inclination angle. In addition, it can be observed that the enhancement in the natural frequency values is larger at higher aspect ratio. For example, the natural frequency value increases approximately by $0.18 \%$ (from 23.104 for $\theta=0^{\circ}$ to 23.146 for $\theta=60^{\circ}$ ) at aspect ratio $=20$ while its value increases by $5.2 \%$ (from 22.223 for $\theta=0^{\circ}$ to 23.380 for $\theta=60^{\circ}$ ) at aspect ratio $=50$. Additionally, the results in Fig. 7 show an interesting phenomenon. When the aspect ratio equals 50 and the inclination angle is in the range of 40-90, the influence of the inclination angle on the fundamental natural frequencies becomes higher than the influence of the aspect ratio on the fundamental natural frequencies, i.e., the increase of inclination angle compensates the loss in natural frequency value due to the increase of aspect ratio.

Also, it can be observed from Fig. 7 that the natural frequency of the pipe has an increasing trend up to the inclination angle of $\theta=90^{\circ}$ and a reverse trend afterward, i.e., beyond the angle $90^{\circ}$ the behavior is reflected. This can be explained as follows: for an inclined pipe, the gravitational force generated by the weight of the pipe and the fluid is divided into two components, the lateral component and the axial component relative to the pipe axes. When the angle of inclination of the pipe is $\leq 90^{\circ}$, the axial component exerts a tensile force on the pipe, which increases with the increase of the inclination angle of the pipe and the aspect ratio. This in turn reduces the impact of the lateral component, responsible for the occurrence of the lateral displacement of the pipe, and thus increases natural frequencies of the inclined pipe. On the contrary, as the pipe's inclination angle $\theta>90^{\circ}$, the axial component gradually decreases and the lateral component becomes larger with the increase of the inclination angle which causes the natural frequency become lower and eventually same as the horizontal case.

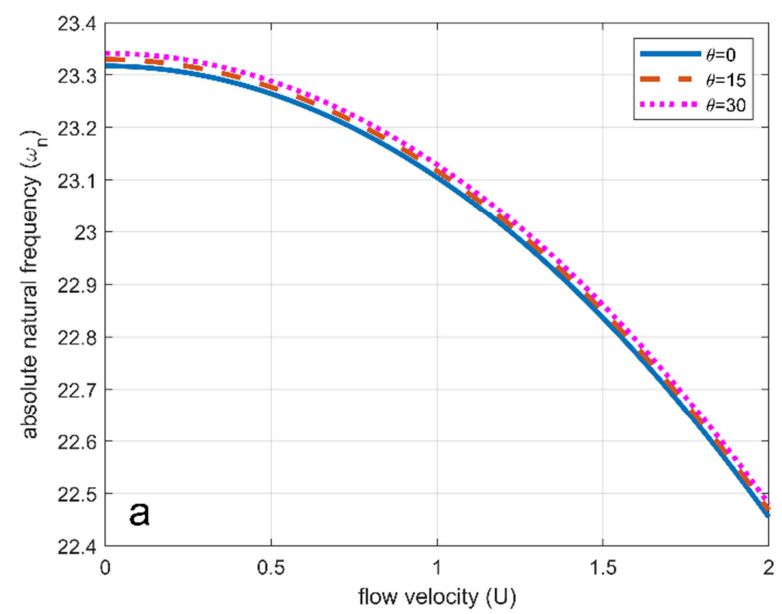

(a) $L / D=20$

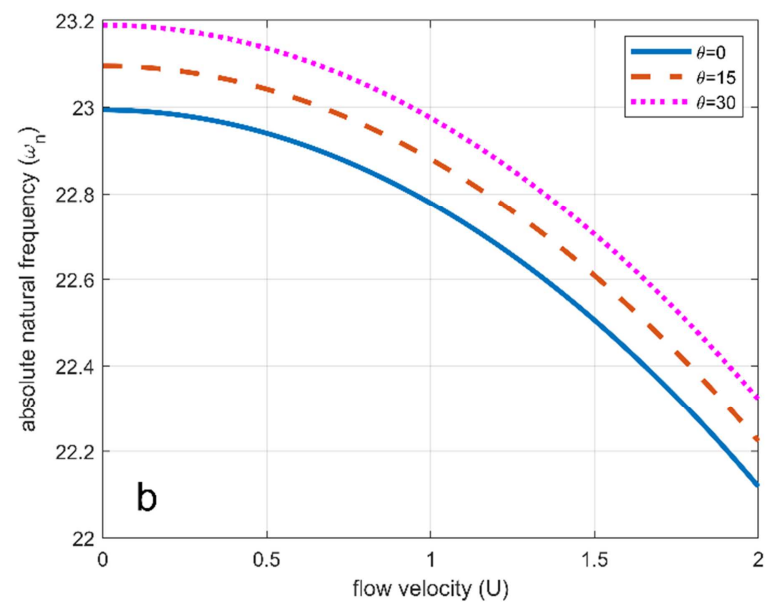

(b) $L / D=30$

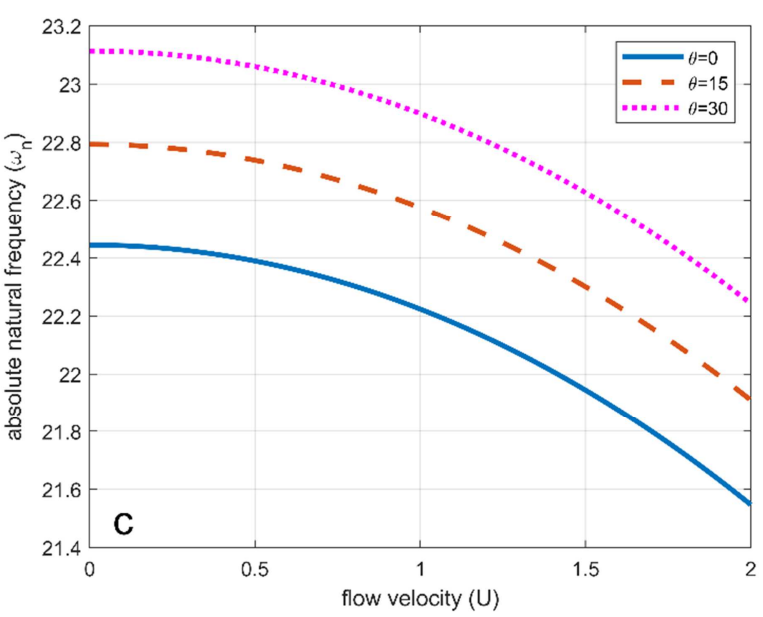

(c) $L / D=50$

Fig. 6 The natural frequency value versus internal fluid velocity with different inclination angles and aspect ratios for the case of $T=25^{\circ} \mathrm{C}$ and $n=1$ 


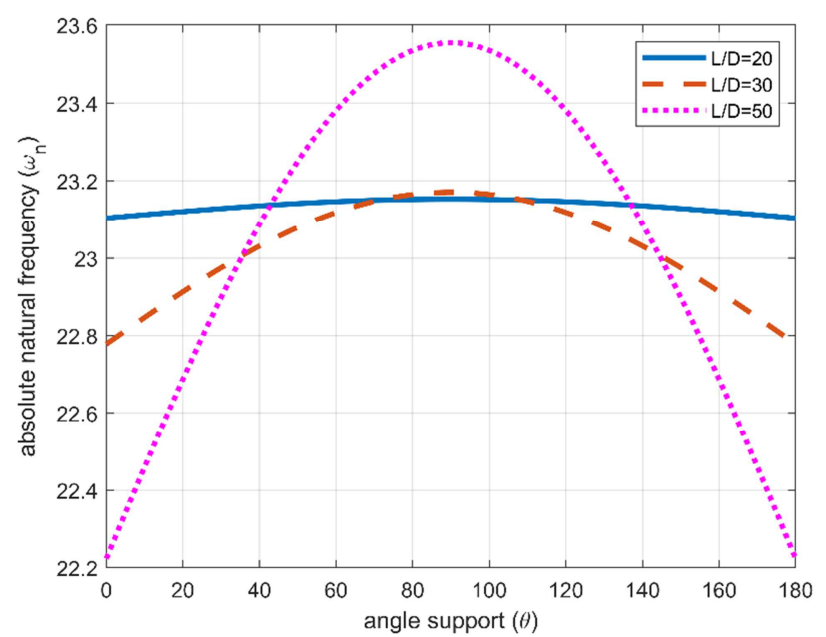

Fig. 7 The natural frequency value versus inclination angle with different aspect ratios for the case of $T=25^{\circ} \mathrm{C}, U=1$, and $n=1$

Fig. 8 describes the effect of the temperature variation on the fundamental natural frequency and the critical velocity of the pipe conveying fluid for different aspect ratios. The results reveal that the temperature variation has a great influence on the fundamental natural frequencies and the critical velocity. Here, as temperature increases, a significant reduction in fundamental natural frequency and critical velocity is observed. Thus, the temperature variations have a softening effect on the pipe stiffness, which is consistent with the work of Ashley et al. [32] where the natural frequency was found to be inversely proportional to the temperature and with the work of Orolu et al. [33] where the critical velocity reduces with the increase of temperature. Also, it is noted that the temperature effect on the fundamental natural frequency and the critical velocity of the pipe conveying fluid is considerably growing at higher aspect ratio. For example, the natural frequency and critical velocity reduce from " 23.23 and 7.46" to " 22.89 and 7.35 " respectively at $L / D=20$, while the lowering is from " 23.2 and 7.38 " to " 4.55 and 0.09 " respectively at $L / D=$ 50. It can be inferred from these results that the pipe may be losing their stability even with zero fluid velocity with the increasing temperature. This suggests that the temperature effect becomes more essential parameter, for engineers and designers, than the velocity of fluid flowing inside the pipe at higher aspect ratio as designing the pipelines systems.

The temperature effect on natural frequencies is further examined by plotting in Fig. 9 the variation of the fundamental natural frequencies with the pipe's inclination angle for different temperatures. The curves in Fig. 9 manifest that when the variation in the pipe temperature is slight, the change of the inclination angle has very little effect on the fundamental natural frequency. However, as the pipe temperature increases, the influence of inclination angle becomes more noticeable.

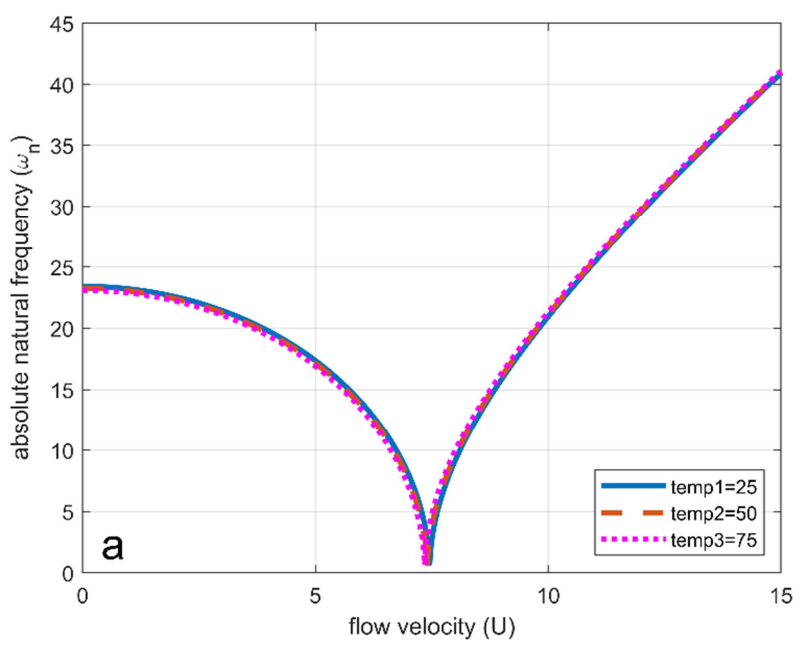

(a) $L / D=20$

Fig. 8 The natural frequency value versus fluid velocity with different temperatures and aspect ratios for the case of $\theta=0^{\circ}$ and $n=1$ 


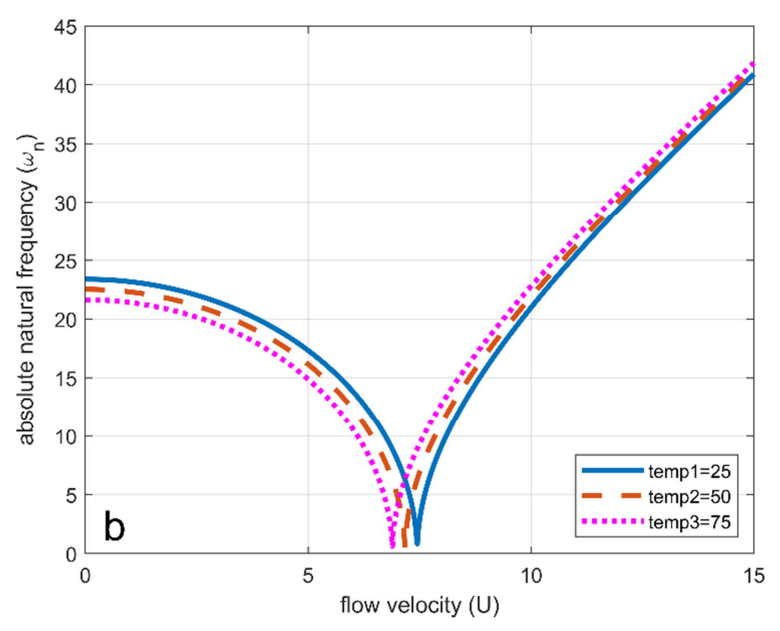

(b) $L / D=30$

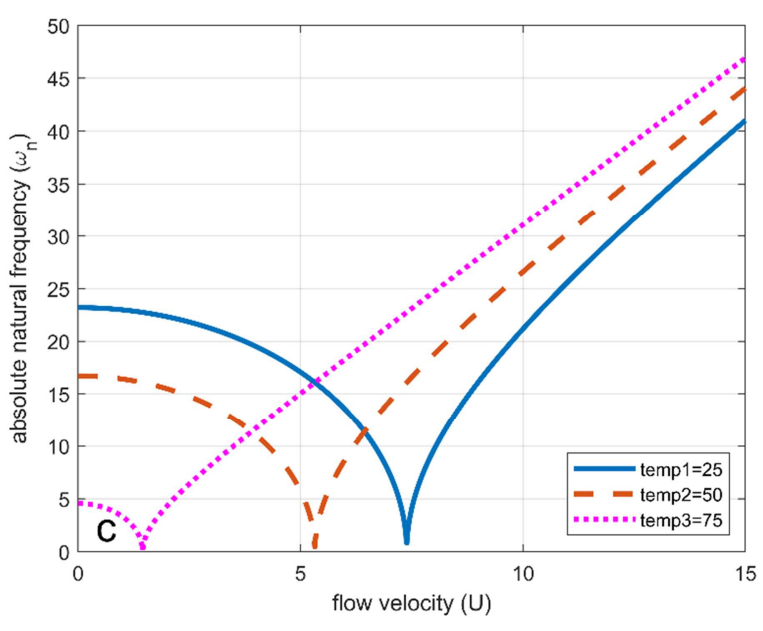

(c) $L / D=50$

Fig. 8 The natural frequency value versus fluid velocity with different temperatures and aspect ratios for the case of $\theta=0^{\circ}$ and $n=1$ (continued)

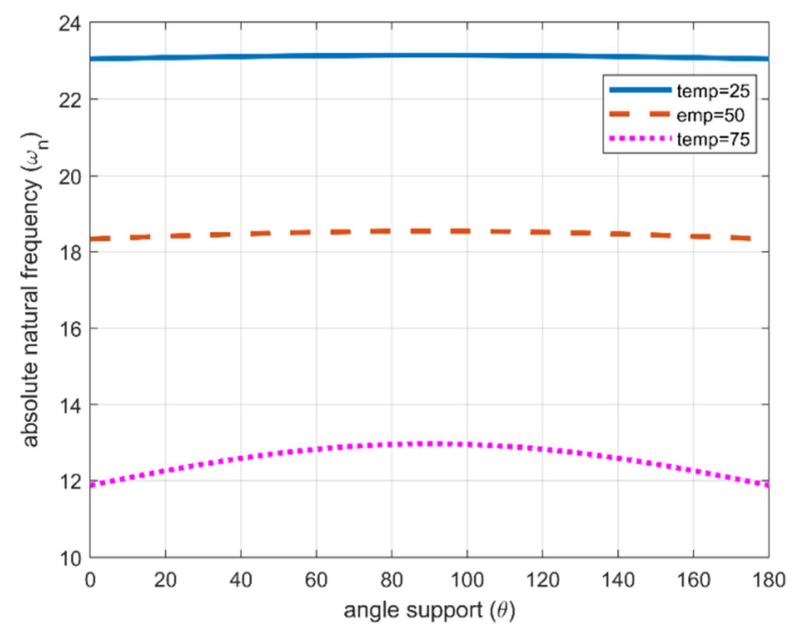

Fig. 9 The natural frequency value versus pipe's inclination angle with different temperatures for the case of $U=1, n=1$, and $L / D=50$

\section{Summary and Conclusions}

The ITT for natural frequency and dynamic response computation of PP-R pipe containing incompressible flowing fluid is proposed in this article. The Euler-Bernoulli beam model is applied to obtain the transverse vibration of the pipe containing flowing fluid. The closed-form analytical expression used to calculate both natural frequency and overall dynamic of the fixed supported pipe containing flowing fluid is derived based on the combination of finite Fourier sine and Laplace transforms and their inverse transform. The effect of various parameters (internal flow velocity, aspect ratio, inclination angle, and temperature variation) on the vibration characteristic of the pipe conveying fluid is presented. The comparison of the numerical results of natural frequency at different internal fluid velocity with the results in published references is conducted and shows a good agreement. The results reveal that the fundamental natural frequency of the pipe containing flowing fluid reduces as the internal flow velocity increases. Once the fundamental natural frequency reduces to zero, the divergence instability happens. The findings of the current study can be summarized as follows:

(1) The proposed analytical method has a clear concept, suitable for hand computation, and gives a theoretical basis for more engineering applications of the inclined fixed supported pipe conveying fluid under thermal loads.

(2) The aspect ratio, temperature variation, and inclination angle strongly affect both the natural frequency and critical velocity of the system. 
(3) There is a strong coupling between the aspect ratio of pipe length to its outside diameter with temperature variation and inclination angle.

(4) The temperature variation is a major concern rather than the internal fluid velocity in the design of the pipe containing flowing fluid at higher aspect ratio.

(5) The inclination angle has larger impact on vibration characteristics at higher aspect ratio, which should be paid attention in engineering.

(6) The natural frequency and critical velocity of the pipe-fluid system decrease while its dynamic deflection increases with the increase of temperature. The divergence can occur even when the fluid velocity equals zero with the increasing temperature of the pipe.

(7) The natural frequency and critical velocity of the pipe conveying fluid decrease while its dynamic deflection increases with the increase of aspect ratio.

\section{Conflicts of Interest}

The authors declare no conflict of interest.

\section{References}

[1] M. P. Païdoussis, Fluid-Structure Interactions: Slender Structures and Axial Flow, 2nd ed., London: Academic Press, 2014.

[2] V. Olunloyo, C. Osheku, and P. Olayiwola, "Concerning the Effect of a Viscoelastic Foundation on the Dynamic Stability of a Pipeline System Conveying an Incompressible Fluid,” Journal of Applied and Computational Mechanics, vol. 2, no. 2, pp. 96-117, 2016.

[3] G. W. Housner, "Bending Vibration of a Pipe Line Containing Flowing Fluid," Journal of Applied Mechanics, vol. 19, no. 2, pp. 205-208, June 1952.

[4] R. A. Stein and M. W. Tobriner, "Vibration of Pipes Containing Flowing Fluids," Journal of Applied Mechanics, vol. 37, no. 4, pp. 906-916, December 1970.

[5] M. P. Païdoussis, "Flutter of Conservative Systems of Pipes Conveying Incompressible Fluid," Journal of Mechanical Engineering Science, vol. 17, no. 1, pp. 19-25, February 1975.

[6] R. H. Plaut and K. Huseyin, "Instability of Fluid-Conveying Pipes under Axial Load," Journal of Applied Mechanics, vol. 42, no. 4, pp. 889-890, December 1975.

[7] F. J. Hatfield, D. C. Wiggert, and R. S. Otwell, "Fluid Structure Interaction in Piping by Component Synthesis," Journal of Applied Mechanics, vol. 104, no. 3, pp. 318-325, September 1982.

[8] M. W. Lesmez, D. C. Wiggert, and F. J. Hatfield, “Modal Analysis of Vibrations in Liquid-Filled Piping Systems,” Journal of Fluids Engineering, vol. 112, no. 3, pp. 311-318, September 1990.

[9] Q. Qian, L. Wang, and Q. Ni, "Instability of Simply Supported Pipes Conveying Fluid under Thermal Loads," Mechanics Research Communications, vol. 36, no. 3, pp. 413-417, April 2009.

[10] D. Zhao, J. Liu, and C. Q. Wu, "Stability and Local Bifurcation of Parameter-Excited Vibration of Pipes Conveying Pulsating Fluid under Thermal Loading," Applied Mathematics and Mechanics, vol. 36, no. 8, pp. 1017-1032, August 2015.

[11] J. K. Kukla, "Application of the Green Functions to the Problem of the Thermally Induced Vibration of a Beam," Journal of Sound and Vibration, vol. 262, no. 4, pp. 865-876, May 2003.

[12] J. R. Blandino and E. A. Thornton, "Thermally Induced Vibration of an Internally Heated Beam," Journal of Vibration and Acoustics, vol. 123, no. 1, pp. 67-75, January 2001.

[13] F. K. Alfosail, A. H. Nayfeh, and M. I. Younis, "Natural Frequencies and Mode Shapes of Statically Deformed Inclined Risers," International Journal of Non-Linear Mechanics, vol. 94, pp. 12-19, September 2017.

[14] C. Gan, S. Jing, S. Yang, and H. Lei, "Effects of Supported Angle on Stability and Dynamical Bifurcations of Cantilevered Pipe Conveying Fluid,” Applied Mathematics and Mechanics, vol. 36, no. 6, pp. 729-746, May 2015.

[15] D. S. Yang and C. M. Wang, "Dynamic Response and Stability of an Inclined Euler Beam under a Moving Vertical Concentrated Load,” Engineering Structures, vol. 186, pp. 243-254, May 2019. 
[16] B. Ram, Engineering Mathematics, India: Pearson Education, 2009.

[17] X. Liang, Z. Wu, L. Wang, G. Liu, Z. Wang, and W. Zhang, "Semianalytical Three-Dimensional Solutions for the Transient Response of Functionally Graded Material Rectangular Plates,” Journal of Engineering Mechanics, vol. 141, no. 9, pp. 1-17, September 2015.

[18] Y. D. Li and Y. R. Yang, "Forced Vibration of Pipe Conveying Fluid by the Green Function Method," Archive of Applied Mechanics, vol. 84, no. 12, pp. 1811-1823, July 2014.

[19] H. B. Wen, Y. R. Yang, P. Li, Y. D. Li, and Y. Huang, "A New Method Based on Laplace Transform and Its Application to Stability of Pipe Conveying Fluid,” Shock and Vibration, vol. 2017, 1472601, 2017.

[20] Z. Lai, L. Jiang, and W. Zhou, “An Analytical Study on Dynamic Response of Multiple Simply Supported Beam System Subjected to Moving Loads," Shock and Vibration, vol. 2018, 2149251, 2018.

[21] X. Tan, H. Ding, and L. Q. Chen, "Nonlinear Frequencies and Forced Responses of Pipes Conveying Fluid via a Coupled Timoshenko Model," Journal of Sound and Vibration, vol. 455, pp. 241-255, September 2019.

[22] L. Jiang, Y. Zhang, Y. Feng, W. Zhou, and Z. Tan, "Dynamic Response Analysis of a Simply Supported Double-Beam System under Successive Moving Loads,” Applied Sciences, vol. 9, no. 10, 2162, May 2019.

[23] W. T. Thomson, Theory of Vibration with Applications, London: Unwin Hyman, 1988.

[24] M. Ali, I. Alshalal, and J. H. Mohmmed, "Effect of the Torsional Vibration Depending on the Number of Cylinders in Reciprocating Engines,” International Journal of Dynamics and Control, vol. 9, pp. 901-909, January 2021.

[25] J. H. Mohmmed, M. A. Tawfik, and Q. A. Atiyah, "Natural Frequency and Critical Velocities of Heated Inclined Pinned PP-R Pipe Conveying Fluid,” Journal of Achievements in Materials and Manufacturing Engineering, vol. 107, no. 1, pp. 15-27, 2021.

[26] Q. Ni, Z. L. Zhang, and L. Wang, “Application of the Differential Transformation Method to Vibration Analysis of Pipes Conveying Fluid," Applied Mathematics and Computation, vol. 217, no. 16, pp. 7028-7038, April 2011.

[27] X. Liang, X. Zha, X. Jiang, L. Wang, J. Leng, and Z. Cao, "Semi-Analytical Solution for Dynamic Behavior of a Fluid-Conveying Pipe with Different Boundary Conditions," Ocean Engineering, vol. 163, pp. 183-190, September 2018.

[28] A. O. Adelaja, "Natural Frequencies of Pressurized Hot Fluid Conveying Pipes," FUOYE Journal of Engineering and Technology, vol. 3, no. 2, pp. 131-135, September 2018.

[29] V. O. Olunloyo, C. A. Osheku, and P. S. Olayiwola, “A Note on an Analytic Solution for an Incompressible Fluid-Conveying Pipeline System,” Advances in Acoustics and Vibration, vol. 2017, 8141523, 2017.

[30] R. H. Plaut, "Postbuckling and Vibration of End-Supported Elastic Pipes Conveying Fluid and Columns under Follower Loads," Journal of Sound and Vibration, vol. 289, no. 1-2, pp. 264-277, January 2006.

[31] V. O. Olunloyo, C. A. Osheku, and S. I. Kuye, "Vibration and Stability Behaviour of Sandwiched Viscoelastic Pipes Conveying a Non-Newtonian Fluid," 29th International Conference on Ocean, Offshore, and Arctic Engineering, pp. 99-111, June 2010.

[32] H. Ashley and G. Haviland, "Bending Vibrations of a Pipe Line Containing Flowing Fluid," Journal of Applied Mechanics, vol. 17, no. 3, pp. 229-232, September 1950.

[33] K. O. Orolu, T. A. Fashanu, and A. A. Oyediran, "Cusp Bifurcation of Slightly Curved Tensioned Pipe Conveying Hot Pressurized Fluid,” Journal of Vibration and Control, vol. 25, no. 5, pp. 1109-1121, March 2019.

Copyright@ by the authors. Licensee TAETI, Taiwan. This article is an open access article distributed under the terms and conditions of the Creative Commons Attribution (CC BY-NC) license (https://creativecommons.org/licenses/by-nc/4.0/). 